\title{
Aging Effect of Temperature Gradients in Li-ion Cells Experimental and Simulative Investigations and the Consequences on Thermal Battery Management
}

\author{
Matthias Fleckenstein ${ }^{1}$, Oliver Bohlen ${ }^{1}$, Bernard Bäker ${ }^{2}$ \\ ${ }^{1}$ BMW Peugeot Citroën Electrification GmbH, Taunusstraße 45, 80807 München, Germany \\ ${ }^{2}$ Department of Vehicle Mechatronics, TU Dresden, Georg-Bähr-Straße 1c, 01069 Dresden, Germany
}

\begin{abstract}
The occurrence and simulative reconstruction of local aging inhomogeneities in Li-ion cells caused by temperature gradients are described, which typically result from active battery cooling in automotive applications. The interactions of thermal, electrical and degradation behaviour are investigated on a prismatic Li-ion cell of 60Ah capacity comprising a graphite / NCM/LMO-blend system. Therefore a transient 3D thermal FVM model combined with a 3D impedance based FNM-model is implemented in order to consider thermal-electrical inhomogeneities. The model is validated by thermal measurements on operated Li-ion cells that are prepared with 8 internal thermocouples inside the jelly roll of the battery. For degradation investigations a weighted charge throughput cycle life model is discretized analogue to the thermal-electrical model. Temperature, current rate and SoC swing are each considered by an aging stress pre-factor. The aging model is exemplary parameterized by five isothermal cycle-life tests. The simulative results show the inhomogeneous capacity loss and impedance rise inside the battery cells with thermal gradient for two aging scenarios. As a result, also temperature and current density distributions inside the cell changes over lifetime. According to both aging indicators, a Li-ion battery cell with temperature gradient comprises an aging relevant temperature that equates the volumetric averaged jelly roll temperature plus $10 \%$ of the maximum temperature difference in the jelly roll.
\end{abstract}

Keywords: Batteries, Modelling, Thermal Management, Li-ion Batteries, Cycle Life

\section{Introduction}

Temperature development within electrical energy storages plays a major role for the aging behaviour of Li-ion cells, which can be seen in several calendar life and cycle life investigations (e.g. $[1,2])$. In modern electrical automotive powertrains, both, the power density and the battery cell sizes of Li-ion cells grow with the progress of technology. Consequently, the maximum temperatures inside battery cells more and more overshoot and decouple from the temperature that can be measured on the surface of the battery cell [3]. In addition, temperature gradients will occur in the jelly roll, which are expected to induce inhomogeneous electrochemical and thermodynamical conditions for the cells aging behaviour [4]. This work demonstrates the effect of thermal electrical inhomogeneities inside Li-ion battery cells on its aging behavior. Therefore, a thermal electrical battery model is introduced and validated. Further on, it is extended by a parametrized three dimensional cycle life aging model. With the combination of all three models local aging differences inside the cell's jelly roll can be detected and the system behavior of the cell over lifetime is evaluated. These examination were operated on a prismatic high energy Li-ion cell type, which is used in battery vehicle powertrains. The specification of the cell is given in table 1. 
Table 1: basic specification of the investigated Li-ion cell

\begin{tabular}{ll}
\hline cell type & $\begin{array}{l}\text { BEV cell } \\
\text { (high energy cell) }\end{array}$ \\
\hline chemistry & NMC / graphite \\
cell geometry & prismatic \\
number of jelly rolls & 4 \\
nominal capacity & $60 \mathrm{Ah}$ \\
nominal OCV & $3.7 \mathrm{~V}$ \\
dimensions / mm & $\mathrm{H}: 125, \mathrm{~L}: 173, \mathrm{D}: 45$ \\
\hline
\end{tabular}

\section{Battery Modeling}

The thermal and electrochemical interdependencies of a Li-ion battery cell make a permanent co-simulation of an electric and a thermal model mandatory to predict the cell's behavior precisely $[6,7]$. In the following two subchapters the combination of a three dimensional thermal $\mathrm{Fi}$ nite Volume Method model (FVM) with a three dimensional electrical Finite Network Method model FNM is introduced.

\subsection{Thermal Modeling}

The thermal model developed in this work is used to simulate the transient temperature distribution inside one Li-ion battery cell. Therefore, the model's input of heat generation is given by the electrical power loss of the jelly roll. By the usage of the Finite Volume Method (FVM) the Fourier Law of heat conductivity [8] is discretized in three dimensions. As the jelly roll of a prismatic battery cell exhibits a more complex geometry than other cell types, the three coordinates of discretization are adapted to the alignment of the electrode layers. Figure 1 shows the geometry and coordinate system of one jelly roll in a prismatic battery cell as well as an exemplary disretization of $4 \times 5 \times 6$ elements.

Herein, $\vec{n}_{\text {circ }}, \vec{n}_{\text {rad }}$ and $\vec{n}_{\text {long }}$ describes the circumferential, the radial and the longitudinal coordinate of the jelly roll. The electrode layer structure of a battery jelly roll can be modelled in thermal simulations by a homogenous material with anisotropic heat conductivity [9]. In the case of a prismatic geometry a high heat conductivity value in circumferential and longitudinal and a low value in radiating direction is assumed. Both values were determined in heat conduction measurements comparable to Maleki et al. [10]. Finally, the linearized temperature development of a volume element (VE) during one time step $\Delta t$ can be described using an explicit numeric solution of the FVM differential equation of:

$$
\begin{aligned}
& T(t+\Delta t)=T(t)+\frac{\Delta t}{\rho V c} . \\
& \cdot\left(\sum_{i=1}^{n} \frac{T_{i}(t)-T(t)}{R_{t h, i}}+\cdot Q_{i r r}+\cdot Q_{r e v}\right)
\end{aligned}
$$
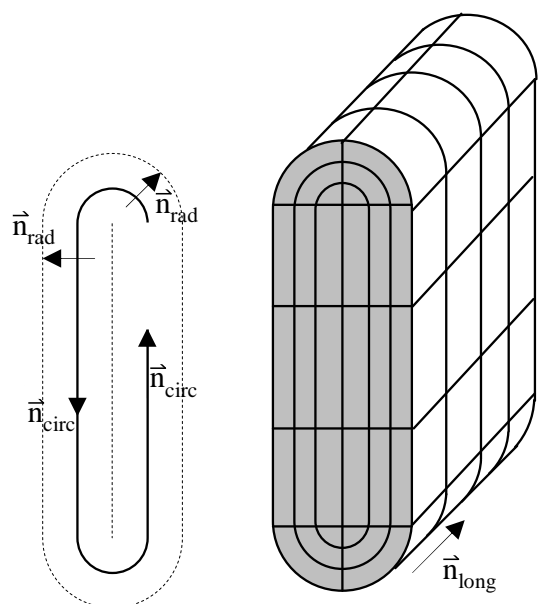

Figure 1: left: Coordinate system for the jelly roll modeling; right: exemplaric discretization of a prismatic jelly roll;

$n$ indicates the number of adjoining volume elements, $T_{i}$ is the temperature of the $i$-th adjoining $\mathrm{VE}$ and $R_{t h, i}$ is the thermal resistance from the VE to the $i$-th adjoining VE. $R_{t h}$ is a geometry dependent value influenced by thermal conductivity.

The cell peripherals - anode and cathode tabs, terminals and housing - are modelled by a network of thermal capacities and resistances. Figure 2 represents schematically the configuration of the discretized thermal battery cell model with four jelly rolls inside.

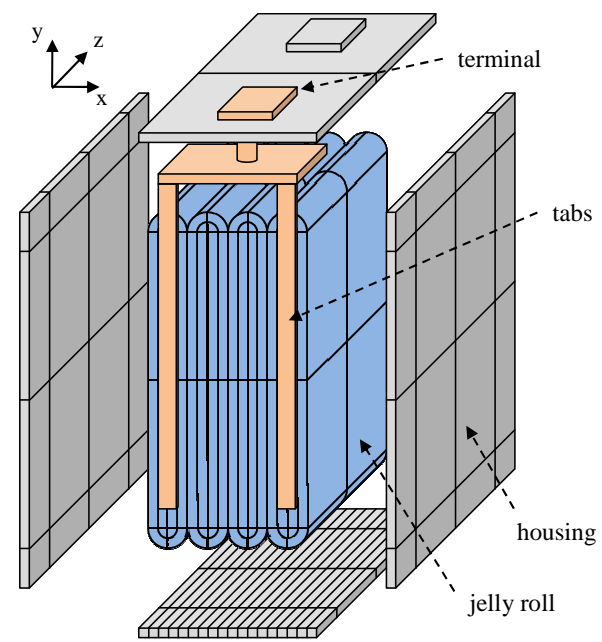

Figure 2: Scheme of the complete thermal battery cell model inclusive battery periphery with reduced discretization

\subsection{Electrical Modeling}

The aim of an electrical battery cell model is the prediction of the electrical cell behaviour, which includes the interdependencies between voltage 
difference of the two cell terminals and the current flow as well as the resulting heat generation inside the jelly roll. Therefore, an equivalent circuit model (ECM) is generated. A review on different complex variations of ECM is given in [11]. In this work, a comparatively simple ECM structure was used as electrical model core that exhibits a serial resistance $R_{S}$ representing the ohmic voltage drop of a battery cell and one RC-circuit (with $R_{P}$ and $C_{P}$ ) representing all transient cell overvoltage effects such as charge transfer, double layer effect and lithium ion diffusion. The low number of circuit elements allows a distinct and fast characterization of battery cells in the time domain. Additionally, the changes of impedance values over lifetime can be determined more precisely as parameter fitting can get ambiguous with a growing number of circuit elements. These benefits outweigh the disadvantage of a slightly lower accuracy compared to more complex models. All parameters of the equivalent circuit are parametrized in dependency of temperature, current direction and SoC. The model core also exhibits an SoC and temperature dependent Open Circuit Voltage (OCV) calculation.

The ECM model core describing the overall battery cell behavior was spatialized to a FiniteNetwork-Method (FNM-) model that enables the determination of a local potential difference, a local charge transfer current density, a local heat generation value and a local SoC for each volume element. For a distinct thermal-electrical cosimulation the FNM-model was discretized according to the thermal model. Thus, each element consists of a node on the cathode current connector and one on the anode current connector as well as a local impedance model and a local OCV source. The impedance parameters of the ECM core were scaled proportionately to the volume fraction of each element in reference to the overall volume of the jelly rolls. An extract of five parallel connected battery cell elements is schematically depicted in figure 3 .

The nodes of each volume element on the cathode current collector are connected to the nodes of the adjoining VEs in circumferential and longitudinal direction by purely ohmic resistances $\left(\mathbf{R}_{\text {circ }, C}\right.$ and $\left.\mathbf{R}_{\text {long,C }}\right)$ that are parametrized by material properties and geometrical values. In analogy, these connections are implemented for the anode nodes $\left(\mathrm{R}_{\text {circ }, A}\right.$ and $\left.\mathbf{R}_{l o n g, A}\right)$. The anode nodes of the longitudinally most outer volume elements are connected to the negative battery terminal. On the opposite side of the battery cell in longitudinal direction the cathode is connected to the positive terminal.

Additionally a linear hysteresis model for determining the local OCV as a function of local SoC and temperature is implemented for each volume element (compare [9]). The discretized battery model determines the current distribution in dependency on the input of the potential difference between the two cell terminals. In order to simulatively load a battery cell by a defined current profile an additional PI-controller was added.

\subsection{Thermal Electrical Validation}

The validation of a combined discretized thermal-electrical battery cell model can be executed in two different ways. One possibility to validate the thermal electrical interdependencies is arranged for example by the current flow analysis of several differently tempered battery cells that are connected in parallel [9]. In this work the development of the temperature distribution inside the jelly rolls are measured directly by eight thermocouples integrated into the battery cell jelly rolls by the cell supplier. All sensor positions are schematically depicted in figure 4.

Internal and External Thermocouples $\mathrm{T}_{\mathrm{I}}$ and $\mathrm{T}_{\mathrm{E}}$

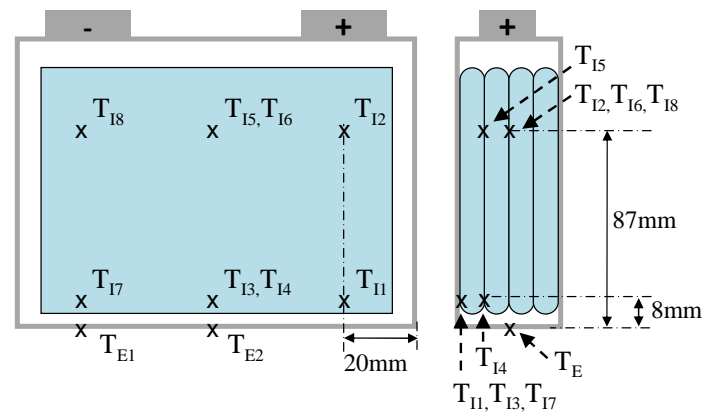

Figure 4: Sensor position layout of the eight internal and two external thermocouples on the Li-ion test cell

The single Li-ion test-cell was placed on two cooled aluminium stripes. Two external temperature sensors $\left(\mathrm{T}_{E 1}\right.$ and $\left.\mathrm{T}_{E 2}\right)$ were placed on the cell bottom between the connection areas to the cooling stripes. All other battery cell surfaces were insulated by $20 \mathrm{~mm}$ thick plastic foams. The tests were operated in a climate chamber with a constant ambient temperature of $20^{\circ} \mathrm{C}$ and a homogenized initial battery temperature of also $20^{\circ} \mathrm{C}$. The battery test cell was connected to an electrical cell test bench. The current load has been defined by a SoC-neutral constant current pulse cycle with $240 \mathrm{~A}$ discharge pulses of $5 \mathrm{~s}$ and $120 \mathrm{~A}$ charge pulses of $10 \mathrm{~s}$ at $50 \%$ SoC. No load breaks were implemented between charge and discharge pulses. The experiment was stopped after three hours, when a thermal steady state was reached.

For the validation of the thermal-electrical battery model the cell was simulated considering the same circumstances as in the test bench and the measured cell bottom temperatures were used as thermal boundary condition of the battery cell.

Table 2 compares the temperature of all thermocouples to the simulated values at the same geometrical position in the thermal steady state. Figure 5 visualizes the comparison of the steady state temperatures by a cross sectional view on the simulated Li-ion cell temperature distribution to the according measurement results. The temperature distribution inside the battery cell exhibits a good accordance to the simulated temperature values. The accuracy tolerance of the cell model was defined as the average absolute temperature difference between sensor and simula- 


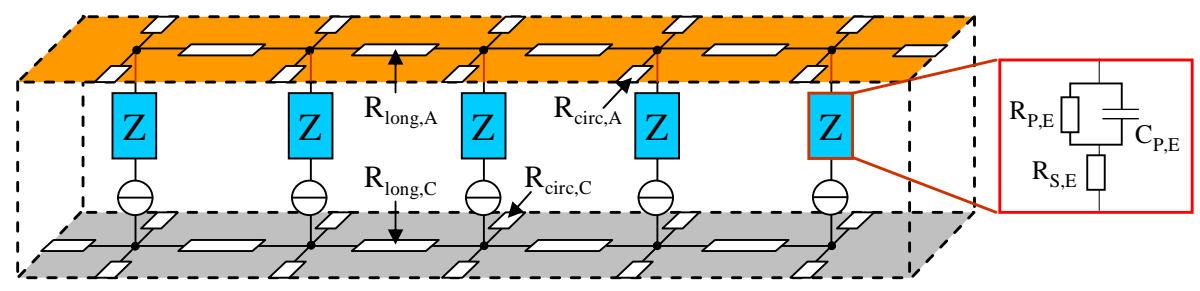

Figure 3: Scheme of five parallel connected battery cell elements composed of two electrode collectors bonded by several model cores

Table 2: Comparison of the internal and external steady state temperatures in the validation test after $9000 \mathrm{~s}$ to the values of simulation

\begin{tabular}{ccc} 
Thermocouple & Measurement & Simulation \\
\hline$T_{E 1}$ & $24.4^{\circ} \mathrm{C}$ & - \\
$T_{E 2}$ & $23.4^{\circ} \mathrm{C}$ & - \\
$T_{I 1}$ & $24.0^{\circ} \mathrm{C}$ & $24.8^{\circ} \mathrm{C}$ \\
$T_{I 2}$ & $32.8^{\circ} \mathrm{C}$ & $33.4^{\circ} \mathrm{C}$ \\
$T_{I 3}$ & $26.5^{\circ} \mathrm{C}$ & $26.3^{\circ} \mathrm{C}$ \\
$T_{I 4}$ & $30.0^{\circ} \mathrm{C}$ & $30.7^{\circ} \mathrm{C}$ \\
$T_{I 5}$ & $32.2^{\circ} \mathrm{C}$ & $32.2^{\circ} \mathrm{C}$ \\
$T_{I 6}$ & $33.6^{\circ} \mathrm{C}$ & $33.5^{\circ} \mathrm{C}$ \\
$T_{I 7}$ & $26.4^{\circ} \mathrm{C}$ & $27.3^{\circ} \mathrm{C}$ \\
$T_{I 8}$ & $34.1^{\circ} \mathrm{C}$ & $34.0^{\circ} \mathrm{C}$
\end{tabular}

tion values in reference to the maximum temperature difference between jelly roll and cell bottom. For the validation test a tolerance during thermal steady state of lower than $4.5 \%(0.4 \mathrm{~K})$ was detected.

Addtionally, figure 6 illustrates the accuracy of the spatialized battery model during thermal dynamic cell load scenarios. Herein, the maximum and minimum jelly roll temperatures of simulation and measurement are displayed over time. Also during the dynamic heat-up phase no bigger temperature differences than $1.0 \mathrm{~K}$ occurred. According to the nominal thermocouple inaccuracy of $1.5 \mathrm{~K}$ the discretized thermal-electrical Li-ion battery model can be observed as validated.

\subsection{Aging Modeling}

In order to predict battery cycle life performance, two basically different aging simulation methods are used in literature. First Principle Models (FPM) are used to mathematically describe the occurrence and velocity of the elementary electrochemical aging reactions inside a battery cell. Therefore, FPM aging models have to be based on an existing physio-chemical battery model representing the charge transfer, migration and diffusion process within the electrodes. These models require a huge degree of knowledge on the design and composition of the battery cell and can often only be used for qualitative evaluations

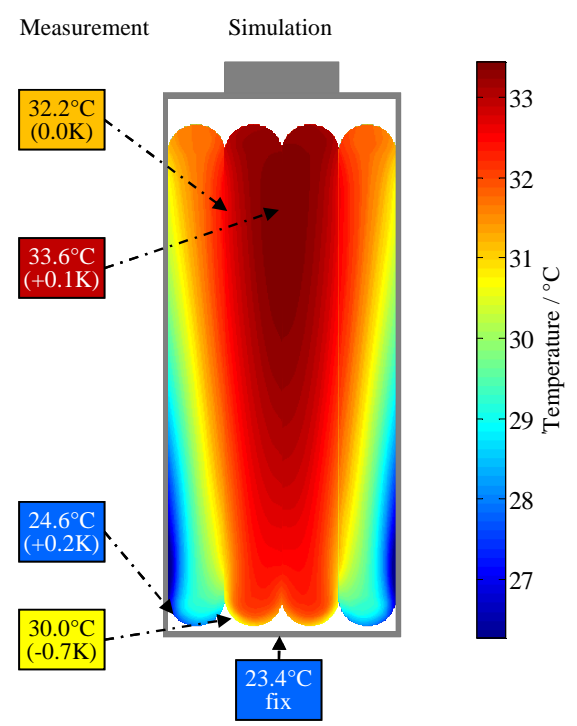

Figure 5: Cross sectional view on the temperature distribution inside the Li-ion cell in the steady state of the validation test and comparison to the measurements of the thermocouples

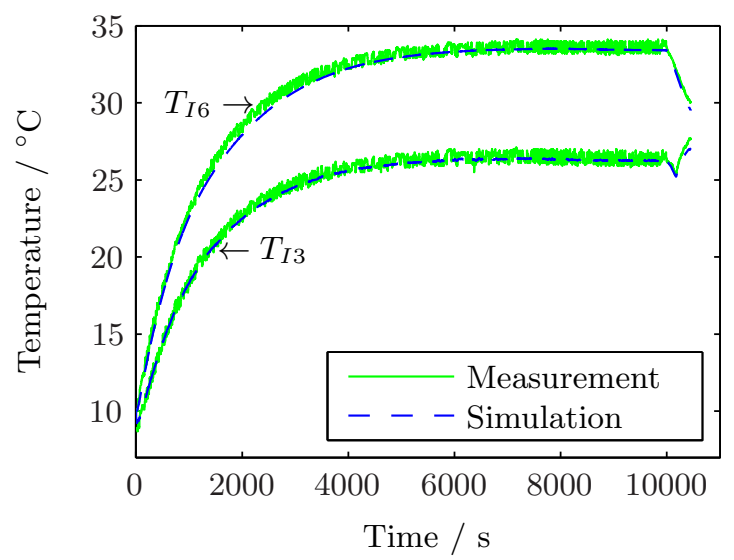

Figure 6: Temperature development of the hottest and coldest internal battery sensor over time in the validation test (green: Measurement, blue: Simulation) 
on basic aging effects in the battery development process.

The specification of the aging model used in this evaluation is not to identify the aging processes and their chemical reaction in detail but to show the impact of thermal electrical inhomogeneities on the system behaviour of the battery cell over lifetime. Thus the second approach of aging simulation by empirical cell degradation models is used. A common way for empirical cycle life aging models is the method of Charge Throughput Models (CTM). The basic assumption of the CTMs is that the state of health $(\mathrm{SoH})$ of a battery continuously decreases with the amount of charge carriers that is transferred from one electrode to the other inside the battery cell over lifetime. According to this method, end of life of a battery cell $(\mathrm{SoH}=1)$ is reached as the defined critical amount of charge throughput has been transferred inside the cell. By the use of a purely CTM, dynamic load scenarios can only be reproduced in an imprecise way neglecting several important battery states and load values influencing the degradation of the battery. Therefore, a more precise approach of empirical aging modeling can be used by amplifying CTMs with weighting factors representing aging stress variables such as the battery temperature. In the exemplary aging model three weighting factors considering the temperature, the current rate and the cycle depth were implemented.

The consideration of the local SoC is an important point in the degradation evaluation of a complete Li-ion cell. But within one cell the SoC difference between two cell elements will rarely rise above 5\% [9], so the SoC dependent weighting factor was neglected in this study.

In order to gain a deeper insight on aging inhomogeneities in Li-ion cells caused by thermal and electrical gradients, the approach of weighted charge throughput models can be locally discretised in three dimensions of the cell's jelly roll. This was directly implemented by the use of the thermal distribution, provided by the discretized thermal model, and the current density and SoC distribution, provided by the discretized electrical model.

Finally, the weighted charge throughput of one battery cell element $C T_{W}(t)$ can be described by integrating the product of the current flow multiplied by the weighting factors for each timestep over lifetime.

$$
C T_{W}(t)=\int_{B o L}^{t}|I(\tau)| \cdot w_{T} \cdot w_{\hat{i}} \cdot w_{\Delta S o C} d \tau
$$

Herein, $w_{T}, w_{\hat{i}}$ and $w_{\triangle S o C}$ are representing the weigthing factors of the cell element's temperature, its current rate and its cycle depth of each charging and discharging period.

\section{Temperature weighting factor $w_{T}$}

According to literature the temperature dependency of degradation processes in li-ion cells can be described by the Arrhenius law of chemistry not only for calendar life but also for cycle life studies $[2,12,13]$. Thus, the velocity of degradation correlates exponentially with the negative inverse of the absolute temperature. For a small temperature range between 0 and $40^{\circ} \mathrm{C}$ compared to the absolute temperature values the dependency can be simplified by the use of Taylor polynomial to an exponential correlation of the degradation with the temperature. Thus, the aging factor $w_{T}$ can be described by

$$
w_{T}=\alpha \cdot e^{\beta \cdot T}
$$

Herein, $\alpha$ is the preexponential aging parameter and $\beta$ the exponential aging parameter of temperature dependency.

\section{Current rate weighting factor $\mathbf{w}_{\hat{i}}$}

Several studies have examined the influence of current rates on the cycle life of Li-ion cells. Thus, not only the growing charge throughput per time but also the growing local over-voltages by higher current rates are expected to tighten the cycle life conditions of battery cells. In Ning et al. [14] a square root dependency of degradation velocity on the current rate were identified for a $\mathrm{Li}$-ion cell with $\mathrm{LiCoO}_{2}$ as cathode material. Anyway, a common power function was implemented in the model for the calculation of the current rate weighting factor.

$$
w_{\hat{i}}=\left(\frac{\hat{i}}{\hat{i}_{\text {ref }}}\right)^{\gamma}
$$

The exponent of current rate dependency is represented by $\gamma$. Therfore, the battery current rate is scaled on the reference current rate $\hat{i}_{\text {ref }}$.

\section{SoC swing weighting factor $\mathbf{w}_{\triangle S o C}$}

During the cycling of battery cells that exhibit electrical inhomogeneities inside, more intensive stressed elements are not only expected to be higher affected of aging mechanism by higher current rates and charge throughput over lifetime. Also the amount of charge throughput per charge/discharge pulse (SoC swing $\triangle S o C$ ) has an influence on the velocity of cell degradation. Even if no mathematically correlation was identified by Wright et al. [13] and Belt et al. [15], herein a positive correlaton between the cycle life degradation and the SoC swing was detected. This can be explained by the growing volume changes caused by the lattice restructuring in the electrodes during longer charge/discharge periods [4]. The weighting factor considering the SoC swing is implemented similar to $w_{\hat{i}}$ as a power function of $\triangle S o C$ normalized to the reference SoC swing. $\epsilon$ represents the exponent of SoC swing dependency.

$$
w_{\Delta S o C}=\left(\frac{\Delta S o C}{\Delta S o C_{r e f}}\right)^{\epsilon}
$$


The weighted charge throughput is utilized as the essential battery aging variable defining the state of health of the Li-ion cell. With the help of this variable, which can be calculated for each battery element, the aging effect indicators, namely capacity loss and impedance rise, can explicitly be determined at any point in time of the battery life and for every battery element.

The changes in impedance over lifetime can be separated in the growing resistive elements $\left(\mathrm{R}_{s}\right.$ and $\left.\mathrm{R}_{p}\right)$ and the decreasing parallel capacity $\left(\mathrm{C}_{p}\right)$ in the electrical equivalent circuit model of the battery. For the calculation of the remaining capacity and the impedance rise, common power functions were chosen. Each of the three equations needs the knowledge of two indicator specific aging parameters $\mathrm{kx} 1$ and $\mathrm{kx} 2$, with $\mathrm{x}$ representing one of the three indicators. Furthermore, the effects of impedance rise and capacity loss can be generated by different cell degradation mechanism (i.e. [4]). Thus, both effects have to be observed decoupled of each other. As a result, the introduced aging model separates between two differently weighted charge throughputs, one value consindering the capacity loss relevant stress factors $\left(C T_{W, C L}\right)$ and one the impedance rise relevant $\left(C T_{W, I R}\right)$.

$$
C_{r e s t}(t)=C_{0} \cdot\left(1-k_{C 1} \cdot C T_{W, C L}(t)^{k_{C 2}}\right)
$$

$$
\begin{aligned}
& R_{x}(t)=R_{x, 0} \cdot\left(1+k_{R 1} \cdot C T_{W, I R}(t)^{k_{R 2}}\right) \\
& C_{P}(t)=C_{P, 0} \cdot\left(1-k_{P 1} \cdot C T_{W, I R}(t)^{k_{P 2}}\right)
\end{aligned}
$$

With $\mathrm{R}_{x}$ representing the serial as well as the parallel resistance of the ECM. For the two resistances, the same aging parameters were used, as the sum of them could be fitted to the parametrizing tests with higher accuracy than the single values.

A complete thermal-electrical simulation of a Liion cell over lifetime considering all aging effects is not feasible in consequence of the 3Ddiscretization. However, the behaviour of Liion cells along long periods of battery aging can be calculated by segmenting the continuous aging process in discrete steps. During one aging step the reference cycle has to be simulated several times until a thermal quasi steady state is reached. The thermal electrical distribution of the last calculated reference cycle is assumed to be representative for 400 reference cycles defining one aging step. Thus, the weighted charge throughput distribution of one aging step equates the charge throughput of its steady state cycle multiplied by 400 (compare to EDLC lifetime modeling in [5]). After determining the two weighted charge throughput distributions per aging step, the impedance rise and remaining capacity distribution for the following aging simulation step can be calculated.

\section{Parametrization tests}

In this work, the aging model isn't parametrized completely in detail with the consideration of all detectable interactions between the aging influence parameters. Nevertheless, it is here parametrized for selective operating points in order to examine and understand the consequences of thermal electrical inhomogeneities on the aging effects in Li-ion cells. Therefore, an exemplary aging relevant load profile was defined as aging reference cycle.

The reference cycle should not contain complete discharge and charge phases, as the operation of cells at SoCs near $0 \%$ and $100 \%$ could cause complex aging mechanism in consequence of local over- and undervoltage, which are mostly independent of electrical inhomogeneities and thus disturb the examination results. It should also be SoC-neutral and, for parametrization, explicitly assignable to one temperature and one current rate. Hence, the reference cycle was specified as a constant current pulse cycle of $1 \mathrm{C}$ for charge and discharge periods at $25^{\circ} \mathrm{C}$ ambient temperature. No resting time was implemented during the change of current direction. The SoC-swing is held between 37.5 and $62.5 \%$ SoC.

For the aging model parametrization the influence of separately varied aging factors (temperature, charge current rate, SoC swing) in the reference cycle are examined in additional cycle life tests. Table 3 gives an overview on all operated cycle life aging tests.

All cells operated in the cycle life tests were first characterized by two full cycles with $0.33 \mathrm{C}$ constant current rate at $20^{\circ} \mathrm{C}$ ambient temperature to determine the battery cell discharge capacity. The characterization cycle continued with $1 \mathrm{C}$ and $4 \mathrm{C}$ discharge current pulses of $30 \mathrm{~s} \mathrm{sep-}$ arated each time by a recharging pulse of $0.33 \mathrm{C}$ and 2 minutes relaxation time in order to determine the impedance rise of the cells. The discharge current pulses were operated at 20,50 and $90 \%$ SoC. After completing the characterization test, the cells were tempered to testing temperature and continuously cycled 320 times (245 times respective, see table 3 ) with the defined current profile. After these approximately 7 days the cells were characterized again. The procedure was repeated, until the defined end of life criteria according to capacity loss was reached $\left(C_{E o L}=0.8 \cdot C_{B o L}\right)$. All tests were performed by AVL List GmbH in Graz.

To examine the cell performance over lifetime, on the one hand the mean values of the measured charge capacities in the first and second full charge/discharge profile during the characterization test were monitored. On the other hand the sum of serial and parallel resistance values, determined by pulse fitting on the $4 \mathrm{C}$ discharge pulse at $50 \%$ SoC, was used for impedance rise modeling. Figure 7 compares the cycle life test results of test T1 to T5. The upper diagrams show the relative capacity loss and impedance rise of the tests needed for the temperature influence examination. The middle diagrams compare the aging curves of the tests with varying charge current 
Table 3: Specification of the operated cycle life aging tests for the parametrization of the aging model

\begin{tabular}{|c|c|c|c|c|c|c|c|c|}
\hline 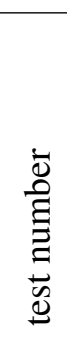 & 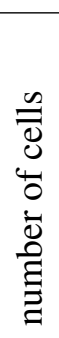 & 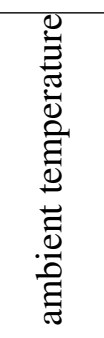 & 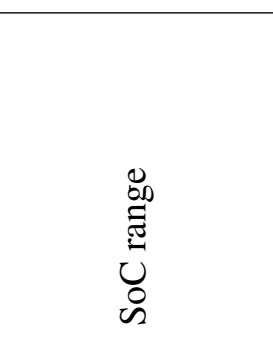 & 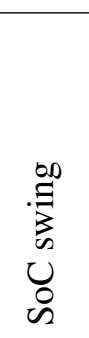 & 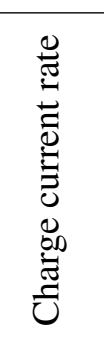 & 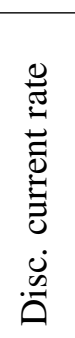 & 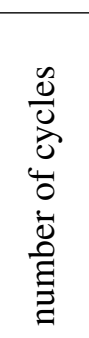 & 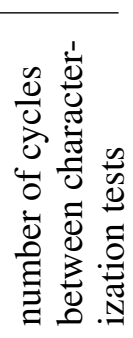 \\
\hline$T 1$ & 2 & $20^{\circ} \mathrm{C}$ & $37.5 \% \leftrightarrow 62.5 \%$ & $25 \%$ & $1 C$ & $1 C$ & 6000 & 320 \\
\hline$T 2$ & 2 & $40^{\circ} \mathrm{C}$ & $37.5 \% \leftrightarrow 62.5 \%$ & $25 \%$ & $1 C$ & $1 C$ & 6000 & 320 \\
\hline$T 3$ & 2 & $20^{\circ} \mathrm{C}$ & $25 \% \leftrightarrow 75 \%$ & $50 \%$ & $1 C$ & $1 C$ & 3000 & 320 \\
\hline$T 4$ & 2 & $20^{\circ} \mathrm{C}$ & $37.5 \% \leftrightarrow 62.5 \%$ & $25 \%$ & $0.5 C$ & $1 C$ & 4500 & 245 \\
\hline$T 5$ & 2 & $20^{\circ} \mathrm{C}$ & $37.5 \% \leftrightarrow 62.5 \%$ & $25 \%$ & $1.5 C$ & $1 C$ & 6000 & 320 \\
\hline
\end{tabular}

rates, the lower diagrams show the test results of the cells with varying SoC swing. All diagrams contain the measurement results as markers at each characterization test and the fitted aging model (equation 2 to 8) as dashed line. All aging model parameters, which were determined by a parallel fitting procedure minimizing a single global residuum, can be seen in table 4.

Comparing test T1 and T2 (figure 7 top), the expected Arrhenius law can be seen with an accelerated capacity loss and impedance rise for $40^{\circ} \mathrm{C}$ ambient temperature. The relative impedance rise seems to be more heavily dependent on the battery temperature. This can be declared, as impedance rise is mostly influenced by the growth of passivation layers on the electrodes that is highly temperature dependent (i.e. [16]). This represents also the need of two differently weighted charge throughputs for aging calculation (compare to section 2.4).

The different aging curves of $\mathrm{T} 1, \mathrm{~T} 4$ and $\mathrm{T} 5$ in the middle diagrams of Figure 7 represent the charge current rate dependency of the reference cycle life aging. Contrary to the expectations a faster cell degradation with growing charge throughput can be consistently seen for lower charge current rates. This arises in the curves of both aging indicators. Anyway, the current rates of $0.5,1$ and $1.5 \mathrm{C}$, which are by far smaller than the charge current limits of the cell at $20^{\circ} \mathrm{C}$, seem not to cause critical current densities for the aging behaviour of the cell. Instead, a longer test time is required for lower current rates to reach the same amount of charge throughput. As a result, the influence of superposed calendar life aging on cycle life grows with declining charge current rates. In the case of $\mathrm{T} 1, \mathrm{~T} 4$ and $\mathrm{T} 5$ the calendar life effect even predominates the aging influence of growing charge current rates. Following, a negative value of the charge current rate exponent $\gamma$ occurs in the model parameterization. The described effect is expected to appear not only for differently cycled battery cells, but qualitatively also between differently loaded jelly roll elements inside one battery cell during cycling. The negative exponent $\gamma$ should not been interpreted as aging acceleration factor for higher current densities, but as correction factor considering the correlation of charge throughput and time.

The two lower diagrams in figure 7 compare the aging behaviours of the Li-ion cells of test 1 and 3 that only differ from each other in the SoC swing amplitude. As both tests do have the same current rate, both tests exhibit the same ratio of charge throughput to test time. With a rising SoC swing from 25 to $50 \%$ only a slow acceleration of aging occurs, which can be identified by the low value of the SoC swing exponent $\epsilon$. These results indicate that in the observed SoC range no significant additional changes in the lattice structure take place with the rise of the SoC swing amplitude.

\section{Results}

Several results that are mentioned in this chapter are specified by exact values. This degree of accuracy refers to the simulation, it cannot be guaranteed for the physical values. Nevertheless, the point of interest should be focused on the qualitative behavior that can be expected for Li-ion cells in common in a wide range of varied simulation input parameters.

The selectively parametrized cycle life model was simulated in different aging scenarios, in order to investigate the effects of thermal electrical inhomogeneities in Li-ion cells on battery aging. On one hand a close to real life aging scenario AS1 was chosen, for which the thermal and electrical gradients inside the battery cell occurs in consequence of self heating caused by the irreversible ohmic power loss. Herein, the $25 \%$ SoC swing cycle with an average SoC of $50 \%$ and a constant current rate of $2 \mathrm{C}$ was selected. A bottom temperature of the cooled cell housing of constantly $20^{\circ} \mathrm{C}$ was chosen as thermal boundary condition. The further cell housing surfaces are modelled as insulated adiabatic walls.

On the other hand, the aging behaviour in a thermal worst case scenario was observed by the aging scenario AS2. Herein, the battery cell 

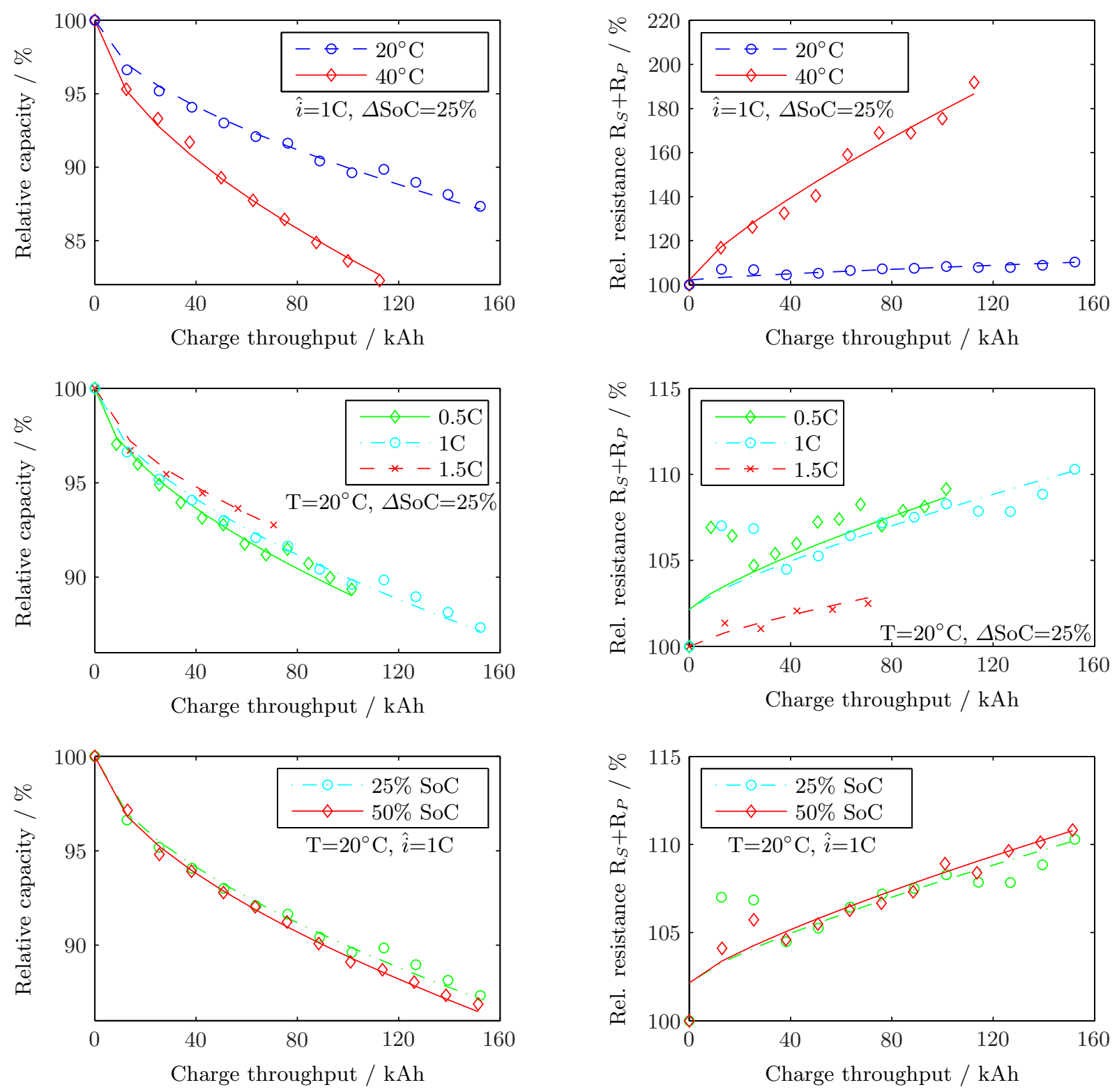

Figure 7: Development of the aging indicators capacity loss and impedance rise over the lifetime charge throughput in the cycle life test 
Table 4: parameters of the aging model determined by the operated cycle life tests 1 to 5

\begin{tabular}{cccccccccc}
\hline \multicolumn{2}{c}{ capacity } & \multicolumn{2}{c}{ impedance } & \multicolumn{2}{c}{ temperature } & \multicolumn{2}{c}{ current rate } & \multicolumn{2}{c}{ SoC swing } \\
\hline$k_{c, 1}$ & $1.2 \cdot 10^{-3}$ & $k_{R, 1}$ & $6.6 \cdot 10^{-6}$ & $\alpha_{c}$ & $6.8 \cdot 10^{-6}$ & $\hat{i}_{r e f}$ & $60 A$ & $\Delta S o C_{r e f}$ & $25 \%$ \\
$k_{c, 2}$ & 0.586 & $k_{R, 2}$ & 0.789 & $\beta_{c}$ & 0.164 & $\gamma$ & -0.196 & $\epsilon$ & 0.13 \\
& & $k_{C_{P}, 1}$ & $4.9 \cdot 10^{-6}$ & $\alpha_{z}$ & $1.3 \cdot 10^{-21}$ & & & & \\
& & $k_{C_{P}, 2}$ & 0.800 & $\beta_{z}$ & $4.1 \cdot 10^{-2}$ & & & & \\
\hline
\end{tabular}

was loaded by the reference current profile (SoC swing: $25 \%$, average SoC: $50 \%$, current rate: $1 \mathrm{C})$. Additionally, the thermal gradient inside the cell is generated by a forced temperature difference between the two large side walls of the prismatic battery cell. One side was assumed to be at a temperature of $40^{\circ} \mathrm{C}$ constant over lifetime, the opposite side wall is forced to stay at $10^{\circ} \mathrm{C}$. This aging scenario can be understood as a case of an inappropriate battery design with an asymmetrical heat flux inside the battery. In reality a similar heat insertion can for example be caused by an asymmetric and unintentional connection between the hot exhaust pipe of a hybrid electrical car and the battery pack cooling device.
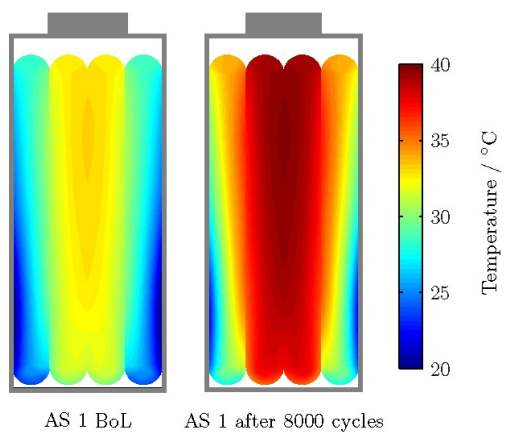

AS 1 BoL

AS 1 after 8000 cycles
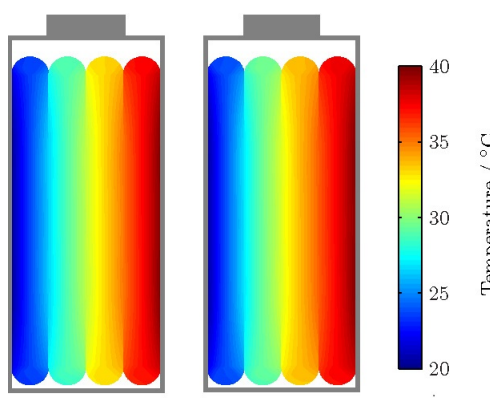

AS $2 \mathrm{BoL}$

AS 2 after 8000 cycles

Figure 8: Temperature distribution inside the jelly roll of the Li-ion cell for aging scenario AS1 and AS2 at Begin and End of Life

The temperature distributions at $\mathrm{BoL}$ and $\mathrm{EoL}$ for both scenarios are shown in figure 8. For AS2 no big difference in the thermal behaviour occurs over lifetime, as the temperature distribution mainly depends on the constant boundary conditions at the side walls. Thereby, a large heat flux through the cell arises compared to its self heat generation. Contrary, the temperature gradient of AS1 is mostly induced by the self heating of the jelly roll. In consequence, a growth of impedance increases the power loss until EoL as well as the absolute temperature and the temperature gradient inside the cell.

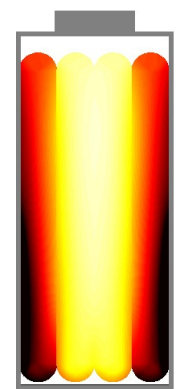

AS 1 BoL

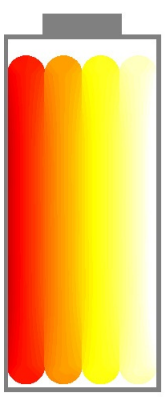

AS 2 BoL

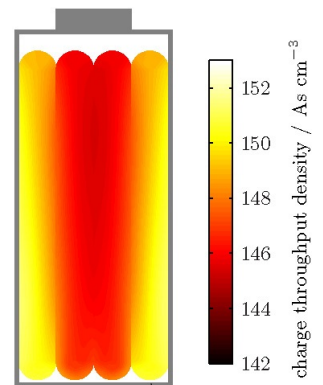

AS 1 after 8000 cycles

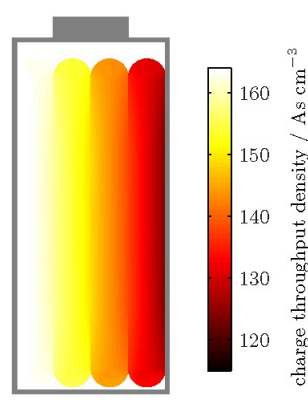

AS 2 after 8000 cycles
Figure 9: Current density distribution inside the jelly roll of the Li-ion cell for aging scenario AS1 and AS2 at Begin and End of Life

The temperature gradient inside the jelly roll generates an impedance gradient for both scenarios at begin of life. A current density gradient occurs form hotter elements with higher current rates to the colder elements with lower current rates. This can be seen for the SoC neutral reference cycle in the two different aging scenarios in figure 9 (top left and down left). As the hotter elements suffer from higher temperatures, higher SoC swing amplitudes and higher charge throughput, an accelerated aging of these elements can be assumed that affects faster impedance rise and capacity loss. As a consequence, the impedance gradient caused by aging inhomogeneities equalizes and finally counterbalances the impedance differences caused by temperature gradients over lifetime. These aging inhomogeneities in the advanced battery life evoke significant electrical inhomogeneities even in nearly isothermal cell load scenarios. Fig- 
ure 9 (top right and down right) demonstrate the inverted current density distribution at end of life. For AS1 the current density ration between the hottest and the coldest cell element starts at 1.15 and continuously decreases down to 0.96 at EoL according to simulation. For the extreme scenario AS2 the current density ratio decreases even from 1.28 to 0.74 . The lower charge throughput for warmer elements of aged battery cells induces a counteracting force of aging inhomogeneity.

Not only the local impedance rise, but also the local capacity loss diverges from BoL in consequence of temperature, SoC swing and charge throughput distributions. Nevertheless, its temperature dependency is not as severe as for the impedance rise. As a result, the capacity loss distribution inside the cell is homogenized in a higher degree than the impedance rise distribution. Figure 10 compares the locally distributed relative impedance rise and relative remaining capacity for AS1 and AS2 at EoL. According to simulation, the local impedances for AS1 after 8000 cycles range from 120 to $144 \%$ of the BoLvalues, the local remaining capacities vary only between 79.6 and $82.6 \%$ of the BoL-values. The tougher aging scenario AS 2 even causes local impedance rises between 112 and $236 \%$ and remaining capacities of 74.1 to $83.9 \%$ inside the jelly roll.

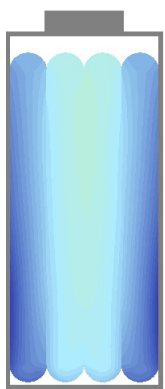

AS 1

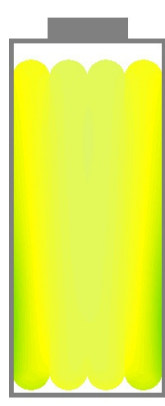

AS 1

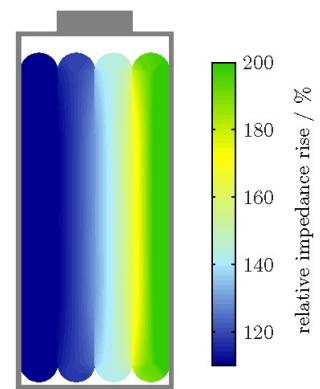

AS 2

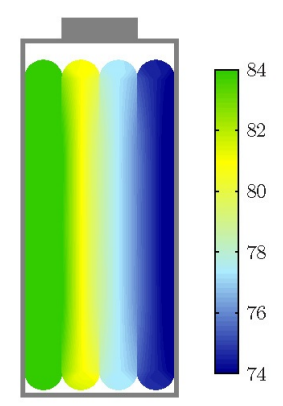

AS 2
Figure 10: Relative impedance and relative remaining capacity distribution inside the jelly roll of the Li-ion cell for aging scenario AS1 and AS2 at EoL

The local distribution of aging effects plays a minor role for the thermal management of Li-ion batteries in electrical powertrains. Instead, the cumulative behaviour of all jelly roll elements describes the remaining power and energy provision of the aged battery cell.

Therefore, the remaining capacities of all cell elements can be added up for each aging step in lifetime to gain the batteries overall remaining capacity. Additionally, the global cell impedance can be determined via the inverse sum of the local jelly roll impedances. For the realistic aging scenario AS1 the capacity loss and impedance rise of one cell over lifetime can be seen in figure 11 . For a better comparability, the aging curves of the thermal inhomogeneous cell are depicted in each case next to the curves of fictive isothermal cells stressed by the same electrical load. One of these fictive cells exhibits consistently the maximum temperature of the inhomogeneous cell $\left(\mathrm{T}_{\max }{ }^{-}\right.$ cell), one cell exhibits consistently the minimum temperature $\left(\mathrm{T}_{\min }\right.$-cell) and the third exhibits the same volume averaged temperature as the inhomogeneous tempered battery cell $\left(\mathrm{T}_{\text {mean }}\right.$-cell). For both aging indicators, the temperature gradient inside the cell does not contribute to an additional aging acceleration at least in the examined range of operation conditions. The impedance rise and the capacity loss curves of the thermal inhomogeneous cell run between the curves of the $\mathrm{T}_{\max }$ - and the $\mathrm{T}_{\min }$-cell. Furthermore, the overall aging velocity of the Li-ion cell with a temperature gradient inside behaves similar to that of the isothermal cell with the same volumetric average temperature.
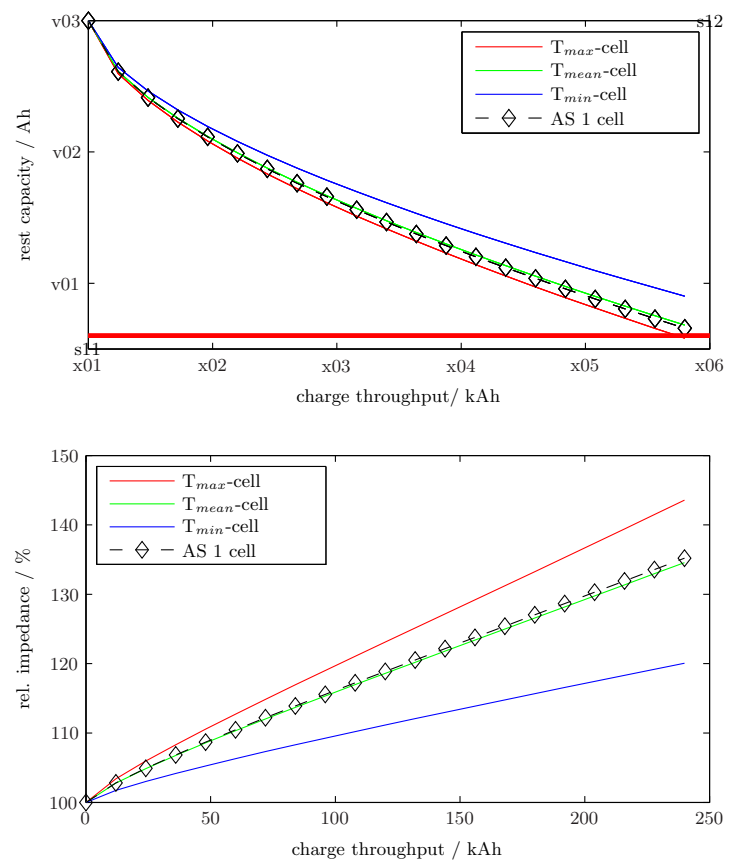

Figure 11: Capacity loss and impedance rise of the Li-ion cell for aging scenario AS1 over cycle lifetime

In order to further investigate the aging influence of temperature gradients in simulation, the capacity fade and impedance rise curves of a cell are observed that is loaded by the extreme aging scenario AS2 over lifetime (compare figure 12). Again, the cell's behaviour is compared to fictive isothermal cells with the maximum temperature, 
the minimum temperature and the mean temperature of the inhomogeneous battery cell as globally uniform temperature. Herein, the extreme temperature gradient does not significantly accelerate the aging progress inside the cell. Similarly to AS1, the degradation curves of the cell with thermal gradient runs nearly congruent to the isothermal cell of the same mean temperature.

Within the parameter range of the experimental cycle life test conditions, the simulation results indicate that a temperature gradient inside a jelly roll does not mean an intensification of the thermal cycle life load defined by the maximal cell temperature. Furthermore, the actual cell degradation correlates with the aging behaviour of an isothermal battery cell of the same average temperature.
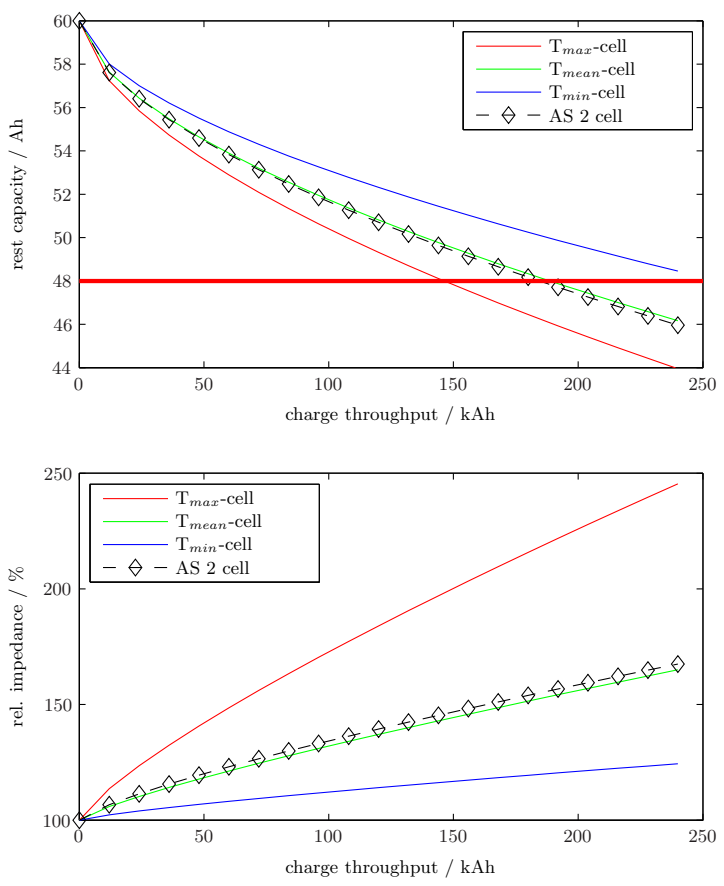

Figure 12: Capacity loss and impedance rise of the Li-ion cell for aging scenario AS2 over cycle lifetime

In consequence of the exponential dependency of aging velocity on the battery temperature (i.e. a low positive value of curvature of the aging velocity against temperature), the aging relevant temperature of an inhomogeneously tempered Li-ion cell lies in simulation marginally above the volumetric mean temperature of the cell.

Finally, an estimation of lifetime considering the maximum battery temperature as homogenous battery temperature is only valid in worst-case scenarios of conservative aging investigations. The use of the volumetric average temperature seems to describe the aging behaviour of a Li-ion cell in a more realistic way. Even so, it underestimates the real aging velocity marginally. The consideration of the minimum jelly roll temperature makes only sense for cycle life investigations in temperature regions, where lithium plating dominates cycle life aging effects (compare to [17]).

Concerning impedance rise, the aging relevant temperature for AS1 exceeds the mean temperature by $0.14 \mathrm{~K}$, which equates a relative offset of $2 \%$ of the occurring maximum temperature difference on the mean temperature of the cell. In analogy, the aging relevant temperature for AS 1 concerning capacity fade is $0.15 \mathrm{~K}(6.1 \%$ of $\left.\Delta T_{\max }\right)$ higher than the average temperature.

Even in the extreme aging scenario, which comprises a temperature difference inside the jelly roll of $30 \mathrm{~K}$, the aging relevant temperature of a battery cell deviates only $5-7 \%$ of the maximum temperature difference from the average temperature of the cell. This is valid for the impedance rise $(7 \%)$ as well as for the capacity loss $(5 \%)$ behaviour. Following, an aging relevant temperature can be deduced for safe but accurate aging observation of the investigated Li-ion cell by:

$$
T_{\text {aging }}=T_{\text {mean }}+10 \% \cdot \Delta T_{\max }
$$

The qualitative results described in this chapter exhibit a high stability on the variation of the aging model parameters. Anyway, their validity is only given, if no additional aging effect occurs in reality that is caused by temperature gradients but is not considered in this work (for example thermal stresses). To the author's knowledge the aging influence of such an effect is not yet mentioned in literature. Nevertheless, cycle life battery tests have to be operated in future that validate the simulation results on inhomogeneously tempered Li-ion cells. Therefore, an externally forced temperature gradient inside the cell similar to the scenario AS2 would be recommended.

\section{Conclusion}

A thermal and electrical three dimensional spatialized battery cell model of a prismatic Li-ion high energy cell is developed. It's accuracy is validated by thermal measurements inside the battery cell during electrical operation. Simulation and experiments do agree with a maximum deviation of $0.4 \mathrm{~K}(4.5 \%)$ during thermal steady state and $1.0 \mathrm{~K}$ during dynamical battery cycles. The thermal-electrical spatialized battery model (developed for BoL cell states) is enlarged by an aging model, also three dimensional spatialized, based on an empirical weighted charge throughput model. The model was parametrized for a $1 \mathrm{C}$ SoC-neutral current reference cycle of $25 \%$ SoC swing at $25^{\circ} \mathrm{C}$ ambient temperature and $50 \%$ SoC. The weighting factors of temperature, current amplitude and SoC swing were determined by cycle life tests with separately varied aging influence factors. The four variations of cycle life test contain increased ambient temperature $\left(40{ }^{\circ} \mathrm{C}\right)$, higher and lower charge current amplitudes $(0.5$ and $1.5 \mathrm{C})$ and an increased SoC drift $(50 \%)$. With the combination of the parametrized empirical aging model and the thermal electrical battery model, the aging influence of temperature gradients inside battery cells within the observed 
parameter range is investigated. Therefore, a realistic aging scenario of a cell with a temperature gradient caused by self heating and an extreme aging scenario, with an externally forced temperature gradient of $30 \mathrm{~K}$ are analyzed. In both scenarios varying current distributions occur over lifetime induced by inhomogeneous impedance rise velocities.

Furthermore, the simulation results of both scenarios indicate an electrical battery performance, which seems to degrade slightly faster than an isothermal battery cell with the same volumetric average temperature. In comparison, an isothermal cell that exhibits the maximum temperature of the cell with a temperature gradient degrades in all observed cases clearly faster than the cell with temperature gradient. For lifetime observation the use of the occurring average temperature as aging relevant temperature is suggested with a correction offset of $10 \%$ of the actual maximum temperature difference in the jelly roll.

\section{References}

[1] I. Bloom et.al., An accelerated calendar and cycle life study of Li-ion cells, Journal of Power sources, 101(2001), 238 - 247.

[2] E.V. Thomas et.al., Statistical methodology for predicting the life of lithium-ion cells, Journal of Power sources, 184(2008), 312-319.

[3] C. Forgez et.al., Thermal modeling of a $\mathrm{LiFePO}_{4}$ /graphite lithium-ion battery, Journal of Power sources, 195(2010), 2961-2968.

[4] J. Vetter et.al., Ageing mechanisms in lithium-ion batteries, Journal of Power sources, 147(2005), 269-281.

[5] O. Bohlen et.al., Ageing behaviour of electrochemical double layer capacitors, Journal of Power sources, 173(2007), 626-632.

[6] W. B. Gu et.al., The use of computer simulation in the evaluation of electric vehicle batteries, Journal of Power sources, 75(1998), 151-161.

[7] V. Srinivasan and C. Y. Wang, Analysis of Electrochemical and Thermal Behavior of Liion Cells, Journal of Electrochemical Society, ISSN 0013-4651, 150(2003), A98-A106.

[8] K. Stephan et al., Thermodynamik - Band1, ISBN 978-3-540-92894-2, Berlin, Springer Verlag, 2009.

[9] M. Fleckenstein et.al., Current density and state of charge inhomogeneities in Li-ion battery cells with LiFePO4 as cathode material due to temperature gradients, Journal of Power sources, 196(2011), 4769-4778.
[10] H. Maleki et.al., Thermal Properties of Li-Ion Battery and Components, Journal of the electrochem. society, 146(1999), 947-954.

[11] S. Buller, Impedance-based simulation models for energy storage devices, $\mathrm{PhD}$ thesis, RWTH Aachen, Shaker Verlag, 2003, ISBN 3-8322-1225-6

[12] I. Bloom et.al., Mechanisms of impedance rise in high-power, lithium-ion cells, Journal of Power sources, 111(2002), 152-159.

[13] R. B. Wright et.al., Power and capacity fade resulting from cycle-life testing of Adv. Techn. Development Program Li-ion batteries, Journal of Power sources, 119(2003), 865-869.

[14] G. Ning et.al., Capacity fade study of lithiumion batteries cycled at high discharge rates, Journal of Power sources, 117(2003), 160-169.

[15] J. Belt et.al., A capacity and power fade study of Li-ion cells during life cycle testing, Journal of Power sources, 123(2003), 241-246.

[16] M. Wohlfahrt-Mehren et.al., Capa. and power fade study of Li-ion cells during life cycle testing, Journal of Pow. sources, 127(2004), 58-64.

[17] J. Fan and S. Tan, Studies on Charging Li-Ion Cells at Low Temper., Journal of The Electrochemical Society, 153(2006), A1081-A1092.

\section{Authors}

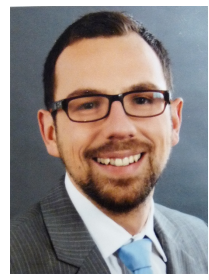

Matthias Fleckenstein was a $\mathrm{PhD}$ student of the TU Dresden at the BMW Group in Munich on the thermal management of Li-ion cells. Since 2012 he works at BMW Peugeot Citroën Electrification as developer of HV Batteries.

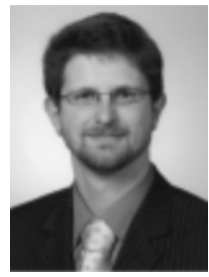

Dr. Oliver Bohlen received his $\mathrm{PhD}$ degree on Impedance Based Battery Monitoring at RWTH Aachen University. He works at BPC Electrification on Functional Development and System Architecture of HV Batteries.

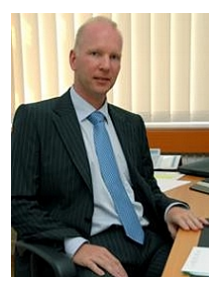

Prof. Dr. Bernard Bäker is chairholder of the department of Vehicle Mechatronics of the TU Dresden. His present research focus ranges from Energy Storages to Driver Assistance Systems and New Vehicle E/E Architectures. 